Article

\title{
Faces of 2-Dimensional Simplex of Order and Chain Polytopes
}

\author{
Aki Mori \\ Department of Pure and Applied Mathematics, Graduate School of Information Science and Technology, \\ Osaka University, Suita, Osaka 565-0871, Japan; u035543c@alumni.osaka-u.ac.jp
}

Received: 11 August 2019; Accepted: 10 September 2019; Published: 14 September 2019

check for updates

\begin{abstract}
Each of the descriptions of vertices, edges, and facets of the order and chain polytope of a finite partially ordered set are well known. In this paper, we give an explicit description of faces of 2-dimensional simplex in terms of vertices. Namely, it will be proved that an arbitrary triangle in 1-skeleton of the order or chain polytope forms the face of 2-dimensional simplex of each polytope. These results mean a generalization in the case of 2 -faces of the characterization known in the case of edges.
\end{abstract}

Keywords: order polytope; chain polytope; partially ordered set

MSC: primary: 52B05; secondary: 06A07

\section{Introduction}

The combinatorial structure of the order polytope $\mathscr{O}(P)$ and the chain polytope $\mathscr{C}(P)$ of a finite poset (partially ordered set) $P$ is explicitly discussed in [1]. Moreover, in [2], the problem when the order polytope $\mathscr{O}(P)$ and the chain polytope $\mathscr{C}(P)$ are unimodularly equivalent is solved. It is also proved that the number of edges of the order polytope $\mathscr{O}(P)$ is equal to that of the chain polytope $\mathscr{C}(P)$ in [3]. In the present paper we give an explicit description of faces of 2-dimensional simplex of $\mathscr{O}(P)$ and $\mathscr{C}(P)$ in terms of vertices. In other words, we show that triangles in 1-skeleton of $\mathscr{O}(P)$ or $\mathscr{C}(P)$ are in one-to-one correspondence with faces of 2-dimensional simplex of each polytope. These results are a direct generalizations of [4] (Lemma 4, Lemma 5).

\section{Definition and Known Results}

Let $P=\left\{x_{1}, \ldots, x_{d}\right\}$ be a finite poset. To each subset $W \subset P$, we associate $\rho(W)=\sum_{i \in W} \mathbf{e}_{i} \in \mathbb{R}^{d}$, where $\mathbf{e}_{1}, \ldots, \mathbf{e}_{d}$ are the canonical unit coordinate vectors of $\mathbb{R}^{d}$. In particular $\rho(\varnothing)$ is the origin of $\mathbb{R}^{d}$. A poset ideal of $P$ is a subset $I$ of $P$ such that, for all $x_{i}$ and $x_{j}$ with $x_{i} \in I$ and $x_{j} \leqslant x_{i}$, one has $x_{j} \in I$. An antichain of $P$ is a subset $A$ of $P$ such that $x_{i}$ and $x_{j}$ belonging to $A$ with $i \neq j$ are incomparable. The empty set $\varnothing$ is a poset ideal as well as an antichain of $P$. We say that $x_{j} \operatorname{covers} x_{i}$ if $x_{i}<x_{j}$ and $x_{i}<x_{k}<x_{j}$ for no $x_{k} \in P$. A chain $x_{j_{1}}<x_{j_{2}}<\cdots<x_{j_{\ell}}$ of $P$ is called saturated if $x_{j_{q}}$ covers $x_{j_{q-1}}$ for $1<q \leqslant \ell$. A maximal chain is a saturated chain such that $x_{j_{1}}$ is a minimal element and $x_{j_{\ell}}$ is a maximal element of the poset. The rank of $P$ is $\sharp(C)-1$, where $C$ is a chain with maximum length of $P$.

The order polytope of $P$ is the convex polytope $\mathscr{O}(P) \subset \mathbb{R}^{d}$ which consists of those $\left(a_{1}, \ldots a_{d}\right) \in \mathbb{R}^{d}$ such that $0 \leqslant a_{i} \leqslant 1$ for every $1 \leqslant i \leqslant d$ together with

$$
a_{i} \geqslant a_{j}
$$

if $x_{i} \leqslant x_{j}$ in $P$. 
The chain polytope of $P$ is the convex polytope $\mathscr{C}(P) \subset \mathbb{R}^{d}$ which consists of those $\left(a_{1}, \ldots, a_{d}\right) \in \mathbb{R}^{d}$ such that $a_{i} \geqslant 0$ for every $1 \leqslant i \leqslant d$ together with

$$
a_{i_{i}}+a_{i_{2}}+\cdots+a_{i_{k}} \leqslant 1
$$

for every maximal chain $x_{i_{1}}<x_{i_{2}}<\cdots<x_{i_{k}}$ of $P$.

One has $\operatorname{dim} \mathscr{O}(P)=\operatorname{dim} \mathscr{C}(P)=d$. The vertices of $\mathscr{O}(P)$ is those $\rho(I)$ for which $I$ is a poset ideal of $P([1]$ (Corollary1.3)) and the vertices of $\mathscr{C}(P)$ is those $\rho(A)$ for which $A$ is an antichain of $P$ ([1] (Theorem2.2)). It then follows that the number of vertices of $\mathscr{O}(P)$ is equal to that of $\mathscr{C}(P)$. Moreover, the volume of $\mathscr{O}(P)$ and that of $\mathscr{C}(P)$ are equal to $e(P) / d$ !, where $e(P)$ is the number of linear extensions of $P$ ([1] (Corollary4.2)). It also follows from [1] that the facets of $\mathscr{O}(P)$ are the following:

- $\quad x_{i}=0$, where $x_{i} \in P$ is maximal;

- $x_{j}=1$, where $x_{j} \in P$ is minimal;

- $x_{i}=x_{j}$, where $x_{j}$ covers $x_{i}$,

and that the facets of $\mathscr{C}(P)$ are the following:

- $x_{i}=0$, for all $x_{i} \in P$;

- $x_{i_{1}}+\cdots+x_{i_{k}}=1$, where $x_{i_{1}}<\cdots<x_{i_{k}}$ is a maximal chain of $P$

In [4] a characterization of edges of $\mathscr{O}(P)$ and those of $\mathscr{C}(P)$ is obtained. Recall that a subposet $Q$ of finite poset $P$ is said to be connected in $P$ if, for each $x$ and $y$ belonging to $Q$, there exists a sequence $x=x_{0}, x_{1}, \ldots, x_{s}=y$ with each $x_{i} \in Q$ for which $x_{i-1}$ and $x_{i}$ are comparable in $P$ for each $1 \leqslant i \leqslant s$.

Lemma 1 ([4] (Lemma 4, Lemma 5)). Let $P$ be a finite poset.

1. Let $I$ and $J$ be poset ideals of $P$ with $I \neq J$. Then the convex hull of $\{\rho(I), \rho(J)\}$ forms an edge of $\mathscr{O}(P)$ if and only if $I \subset J$ and $J \backslash I$ is connected in $P$.

2. Let $A$ and $B$ be antichains of $P$ with $A \neq B$. Then the convex hull of $\{\rho(A), \rho(B)\}$ forms an edge of $\mathscr{C}(P)$ if and only if $(A \backslash B) \cup(B \backslash A)$ is connected in $P$.

\section{Faces of 2-Dimensional Simplex}

Using Lemma 1, we show the following description of faces of 2-dimensional simplex.

Theorem 1. Let $P$ be a finite poset. Let $I, J$, and $K$ be pairwise distinct poset ideals of $P$. Then the convex hull of $\{\rho(I), \rho(J), \rho(K)\}$ forms a 2 -face of $\mathscr{O}(P)$ if and only if $I \subset J \subset K$ and $K \backslash I$ is connected in $P$.

Proof. ("Only if") If the convex hull of $\{\rho(I), \rho(J), \rho(K)\}$ forms a 2-face of $\mathscr{O}(P)$, then the convex hulls of $\{\rho(I), \rho(J)\},\{\rho(J), \rho(K)\}$, and $\{\rho(I), \rho(K)\}$ form edges of $\mathscr{O}(P)$. It then follows from Lemma 1 that $I \subset J \subset K$ and $K \backslash I$ is connected in $P$.

("If") Suppose that the convex hull of $\{\rho(I), \rho(J), \rho(K)\}$ has dimension 1 . Then there exists a line passing through the lattice points $\rho(I), \rho(J)$, and $\rho(K)$. Hence $\rho(I), \rho(J)$, and $\rho(K)$ cannot be vertices of $\mathscr{O}(P)$. Thus the convex hull of $\{\rho(I), \rho(J), \rho(K)\}$ has dimension 2 .

Let $P=\left\{x_{1}, \ldots, x_{d}\right\}$. If there exists a maximal element $x_{i}$ of $P$ not belonging to $I \cup J \cup K$, then the convex hull of $\{\rho(I), \rho(J), \rho(K)\}$ lies in the facet $x_{i}=0$. If there exists a minimal element $x_{j}$ of $P$ belonging to $I \cap J \cap K$, then the convex hull of $\{\rho(I), \rho(J), \rho(K)\}$ lies in the facet $x_{j}=1$. Hence, working with induction on $d(\geqslant 2)$, we may assume that $I \cup J \cup K=P$ and $I \cap J \cap K=\varnothing$. Suppose that $\varnothing=I \subset J \subset K=P$ and $K \backslash I=P$ is connected.

Case 1. $\sharp(J)=1$. 
Let $J=\left\{x_{i}\right\}$ and $P^{\prime}=P \backslash\left\{x_{i}\right\}$. Then $P^{\prime}$ is a connected poset. Let $x_{i_{1}}, \ldots, x_{i_{q}}$ be the maximal elements of $P$ and $\mathcal{A}_{i j}=\left\{y \in P^{\prime} \mid y<x_{i_{j}}\right\}$, where $1 \leqslant j \leqslant q$. Then we write

$$
b_{k}=\left\{\begin{array}{ll}
\sharp\left(\left\{i_{j} \mid x_{k} \in \mathcal{A}_{i j}\right\}\right) & \text { if } k \notin\left\{i_{1}, \ldots, i_{q}, i\right\} \\
0 & \text { if } k=i \\
-\sharp\left(\mathcal{A}_{i j}\right) & \text { if } k \in\left\{i_{1}, \ldots, i_{q}\right\}
\end{array} .\right.
$$

We then claim that the hyperplane $\mathscr{H}$ of $\mathbb{R}^{d}$ defined by the equation $h(\mathbf{x})=\sum_{k=1}^{d} b_{k} x_{k}=0$ is a supporting hyperplane of $\mathscr{O}(P)$ and that $\mathscr{H} \cap \mathscr{O}(P)$ coincides with the convex hull of $\{\rho(\varnothing), \rho(J), \rho(P)\}$. Clearly $h(\rho(\varnothing))=h(\rho(P))=0$ and $h(\rho(J))=b_{i}=0$. Let $I$ be a poset ideal of $P$ with $I \neq \varnothing$, $I \neq P$ and $I \neq J$. We have to prove that $h(\rho(I))>0$. To simplify the notation, suppose that $I \cap\left\{x_{i_{1}}, \ldots, x_{i_{q}}\right\}=\left\{x_{i_{1}}, \ldots, x_{i_{r}}\right\}$, where $0 \leqslant r<q$. If $r=0$, then $h(\rho(J))>0$. Let $1 \leqslant r<q, I^{\prime}=I \backslash\left\{x_{i}\right\}$, and $K=\bigcup_{j=1}^{r}\left(\mathcal{A}_{i_{j}} \cup\left\{x_{i_{j}}\right\}\right)$. Then $I^{\prime}$ and $K$ are poset ideals of $P$ and $h(\rho(K)) \leqslant h\left(\rho\left(I^{\prime}\right)\right)=h(\rho(I))$. We claim $h(\rho(K))>0$. One has $h(\rho(K)) \geqslant 0$. Moreover, $h(\rho(K))=0$ if and only if no $z \in K$ belongs to $\mathcal{A}_{i_{r+1}} \cup \cdots \cup \mathcal{A}_{i_{q}}$. Now, since $P^{\prime}$ is connected, it follows that there exists $z \in K$ with $z \in \mathcal{A}_{i_{r+1}} \cup \cdots \cup \mathcal{A}_{i_{q}}$. Hence $h(\rho(K))>0$. Thus $h(\rho(I))>0$.

Case 2. $\sharp(J)=d-1$.

Let $P \backslash J=\left\{x_{i}\right\}$ and $P^{\prime}=P \backslash\left\{x_{i}\right\}$. Then $P^{\prime}$ is a connected poset. Thus we can show the existence of a supporting hyperplane of $\mathscr{O}(P)$ which contains the convex hull of $\{\rho(\varnothing), \rho(J), \rho(P)\}$ by the same argument in Case 1.

Case 3. $2 \leqslant \sharp(J) \leqslant d-2$.

To simplify the notation, suppose that $J=\left\{x_{1}, \ldots, x_{\ell}\right\}$. Then $P \backslash J=\left\{x_{\ell+1}, \ldots, x_{d}\right\}$. Since $J$ and $P \backslash J$ are subposets of $P$, these posets are connected. Let $x_{i_{1}}, \ldots, x_{i_{q}}$ be the maximal elements of $J$ and $x_{i_{q+1}}, \ldots, x_{i_{q+r}}$ the maximal elements of $P \backslash J$. Then we write

$$
\mathcal{A}_{i j}= \begin{cases}\left\{y \in J \mid y<x_{i_{j}}\right\} & \text { if } 1 \leqslant j \leqslant q \\ \left\{y \in P \backslash J \mid y<x_{i_{j}}\right\} & \text { if } q+1 \leqslant j \leqslant r\end{cases}
$$

and

$$
b_{k}=\left\{\begin{array}{ll}
\sharp\left(\left\{i_{j} \mid x_{i} \in \mathcal{A}_{i j}\right\}\right) & \text { if } k \notin\left\{i_{1}, \ldots, i_{q}, i_{q+1}, \ldots, i_{q+r}\right\} \\
-\sharp\left(\mathcal{A}_{i j}\right) & \text { if } k \in\left\{i_{1}, \ldots, i_{q}, i_{q+1}, \ldots, i_{q+r}\right\}
\end{array} .\right.
$$

We then claim that the hyperplane $\mathscr{H}$ of $\mathbb{R}^{d}$ defined by the equation $h(\mathbf{x})=\sum_{k=1}^{d} b_{k} x_{k}=0$ is a supporting hyperplane of $\mathscr{O}(P)$ and $\mathscr{H} \cap \mathscr{O}(P)$ coincides with the convex hull of $\{\rho(\varnothing), \rho(J), \rho(P)\}$. Clearly $h(\rho(\varnothing))=h(\rho(J))=h(\rho(P \backslash J))=0$, then $h(\rho(P))=h(\rho(J))+h(\rho(P \backslash J))=0$. Let $I$ be a poset ideal of $P$ with $I \neq \varnothing, I \neq P$ and $I \neq J$. What we must prove is $h(\rho(I))>0$.

If $I \subset J$, then $I$ is a poset ideal of $J$. To simplify the notation, suppose that $I \cap\left\{x_{i_{1}}, \ldots, x_{i_{q}}\right\}=$ $\left\{x_{i_{1}}, \ldots, x_{i_{s}}\right\}$, where $0 \leqslant s<q$. If $s=0$, then $h(\rho(I))>0$. Let $1 \leqslant s<q, K=\bigcup_{j=1}^{s}\left(\mathcal{A}_{i_{j}} \cup\left\{x_{i_{j}}\right\}\right)$. Then $K$ is a poset ideal of $J$ and $h(\rho(K)) \leqslant h(\rho(I))$. Thus we can show $h(\rho(K))>0$ by the same argument in Case 1 (Replace $r$ with $s$ and $P^{\prime}$ with $J$ ).

If $J \subset I$, then $I \backslash J$ is a poset ideal of $P \backslash J$. To simplify the notation, suppose that $(I \backslash J) \cap$ $\left\{x_{i_{q+1}}, \ldots, x_{i_{q+r}}\right\}=\left\{x_{i_{q+1}}, \ldots, x_{i_{q+t}}\right\}$, where $0 \leqslant t<r$. If $t=0$, then $h(\rho(I))=h(\rho(J))+h(\rho(I \backslash J))=$ $h(\rho(I \backslash))>0$. Let $1 \leqslant t<r, K=\bigcup_{j=q+1}^{q+t}\left(\mathcal{A}_{i_{j}} \cup\left\{x_{i_{j}}\right\}\right)$. Then $K$ is a poset ideal of $P \backslash J$ and $h(\rho(K)) \leqslant h(\rho(I \backslash J))=h(\rho(I))$. Thus we can show $h(\rho(K))>0$ by the same argument in Case 1 (Replace $r$ with $q+t, q$ with $q+r$ and $P^{\prime}$ with $\left.P \backslash J\right)$. Consequently, $h(\rho(I))>0$, as desired.

Let $A \triangle B$ denote the symmetric difference of the sets $A$ and $B$, that is $A \triangle B=(A \backslash B) \cup(B \backslash A)$.

Theorem 2. Let $P$ be a finite poset. Let $A, B$, and $C$ be pairwise distinct antichains of $P$. Then the convex hull of $\{\rho(A), \rho(B), \rho(C)\}$ forms a 2 -face of $\mathscr{C}(P)$ if and only if $A \triangle B, B \triangle C$ and $C \triangle A$ are connected in $P$. 
Proof. ("Only if") If the convex hull of $\{\rho(A), \rho(B), \rho(C)\}$ forms a 2-face of $\mathscr{C}(P)$, then the convex hulls of $\{\rho(A), \rho(B)\},\{\rho(B), \rho(C)\}$, and $\{\rho(A), \rho(C)\}$ form edges of $\mathscr{C}(P)$. It then follows from Lemma 1 that $A \triangle B, B \triangle C$ and $C \triangle A$ are connected in $P$.

("If") Suppose that the convex hull of $\{\rho(A), \rho(B), \rho(C)\}$ has dimension 1 . Then there exists a line passing through the lattice points $\rho(A), \rho(B)$, and $\rho(C)$. Hence $\rho(A), \rho(B)$, and $\rho(C)$ cannot be vertices of $\mathscr{C}(P)$. Thus the convex hull of $\{\rho(A), \rho(B)$,

$\rho(C)\}$ has dimension 2 .

Let $P=\left\{x_{1}, \ldots, x_{d}\right\}$. If $A \cup B \cup C \neq P$ and $x_{i} \notin A \cup B \cup C$, then the convex hull of $\{\rho(A), \rho(B), \rho(C)\}$ lies in the facet $x_{i}=0$. Furthermore, if $A \cup B \cup C=P$ and $A \cap B \cap C \neq \varnothing$, then $x_{j} \in A \cap B \cap C$ is isolated in $P$ and $x_{j}$ itself is a maximal chain of $P$. Thus the convex hull of $\{\rho(A), \rho(B), \rho(C)\}$ lies in the facet $x_{j}=1$. Hence, working with induction on $d(\geqslant 2)$, we may assume that $A \cup B \cup C=P$ and $A \cap B \cap C=\varnothing$. As stated in the proof of [3] ([Theorem 2.1]), if $A \triangle B$ is connected in $P$, then $A$ and $B$ satisfy either (i) $B \subset A$ or (ii) $y<x$ whenever $x \in A$ and $y \in B$ are comparable. Hence, we consider the following three cases:

(a) If $B \subset A$, then $A \triangle B=A \backslash B$ is connected in $P$, and thus $\sharp(A \backslash B)=1$. Let $A \backslash B=\left\{x_{k}\right\}$. If $C \cap A \neq \varnothing$, then $C \cap A=\left\{x_{k}\right\}$, since $A \cap B \cap C=C \cap B=\varnothing$. Namely $x_{k}$ is isolated in $P$. Hence $B \triangle C=B \cup C=A \cup B \cup C=P$ cannot be connected. Thus $C \cap A=\varnothing$. In this case, we may assume $z<x$ if $x \in A$ and $z \in C$ are comparable. Furthermore, $P$ has rank 1 .

(b) If $B \leftarrow A$ and $B \cap A \neq \varnothing$, then we may assume $y<x$ if $x \in A$ and $y \in B$ are comparable. If $C \subset B$ with $C \cap A \cap B=\varnothing$, then as stated in (a), $C \triangle A$ cannot be connected. Since $C \neq B$, we may assume $z<y$ if $y \in B$ and $z \in C$ are comparable. If $C \cap B \neq \varnothing$, then $C \cap A=\varnothing$ and $P$ has rank 1 or 2 . Similarly, if $C \cap B=\varnothing$, then $C \cap A=\varnothing$ and $P$ has rank 2 .

(c) Let $B \oplus A$ and $B \cap A=\varnothing$. We may assume that if $x \in A$ and $y \in B$ are comparable, then $y<x$. If $C \subset B$, then we regard this case as equivalent to (a). Let $C \not B$. We may assume $z<y$ if $y \in B$ and $z \in C$ are comparable. Moreover, if $C \cap B \neq \varnothing$, then we regard this case as equivalent to (b). If $C \cap B=\varnothing$, then $C \cap A=\varnothing$ and $P$ has rank 2 .

Consequently, there are five cases as regards antichains for $\mathscr{C}(P)$.

Case 1. $B \subset A, C \cap A=\varnothing$, and $C \cap B=\varnothing$.

For each $x_{i} \in B$ we write $b_{i}$ for the number of elements $z \in C$ with $z<x_{i}$. For each $x_{j} \in C$ we write $c_{j}$ for the number of elements $y \in B$ with $x_{j}<y$. Let $a_{k}=0$ for $A \backslash B=\left\{x_{k}\right\}$. Clearly $\sum_{x_{i} \in B} b_{i}=$ $\sum_{x_{j} \in C} c_{j}=q$, where $q$ is the number of pairs $(y, z)$ with $y \in B, z \in C$ and $z<y$. Let $h(\mathbf{x})=\sum_{x_{i} \in B} b_{i} x_{i}+$ $\sum_{x_{j} \in C} c_{j} x_{j}+a_{k} x_{k}$ and let $\mathscr{H}$ be the hyperplane of $\mathbb{R}^{d}$ defined by $h(\mathbf{x})=q$. Then $h(\rho(A))=h(\rho(B))=$ $h(\rho(C))=q$. We claim that, for any antichain $D$ of $P$ with $D \neq A, D \neq B$, and $D \neq C$, one has $h(\rho(D))<q$. Let $D=B_{1} \cup C_{1}$ or $D=\left\{x_{k}\right\} \cup C_{1}$ with $B_{1} \subsetneq B$ and $C_{1} \subsetneq C$. Suppose $D=B_{1} \cup C_{1}$. Since $B \triangle C$ is connected and since $D$ is an antichain of $P$, it follows that $\sum_{x_{i} \in B_{1}} b_{i}+\sum_{x_{j} \in C_{1}} c_{j}<q$. Thus $h(\rho(D))<q$. Suppose that $D=\left\{x_{k}\right\} \cup C_{1}$. It follows that $\sum_{x_{j} \in C_{1}} c_{j}+a_{k}=\sum_{x_{j} \in C_{1}} c_{j}<\sum_{x_{j} \in C} c_{j}=q$. Thus $h(\rho(D))<q$.

Case 2. $B \leftarrow A, B \cap A \neq \varnothing, C \notin B, C \cap B \neq \varnothing, C \cap A=\varnothing$, and $P$ has rank 1 .

We define four numbers as follows:

$$
\begin{aligned}
\alpha_{i} & =\sharp\left(\left\{y \in B \backslash A \mid y<x_{i}, x_{i} \in A \backslash B\right\}\right) ; \\
\gamma_{j} & =\sharp\left(\left\{x \in A \backslash B \mid x_{j}<x, x_{j} \in B \backslash A\right\}\right) ; \\
\alpha_{k} & =\sharp\left(\left\{z \in C \backslash B \mid z<x_{k}, x_{k} \in B \backslash C\right\}\right) ; \\
\gamma_{\ell} & =\sharp\left(\left\{y \in B \backslash C \mid x_{\ell}<y, x_{\ell} \in C \backslash B\right\}\right) .
\end{aligned}
$$


Since $P$ has rank $1, B \subset A \cup C=P$. It follows that $A=(A \backslash B) \cup(B \backslash C), C=(B \backslash A) \cup(C \backslash B)$. Then

$$
\begin{aligned}
\sum_{x_{s} \in A} \alpha_{s}= & \sum_{x_{i} \in A \backslash B} \alpha_{i}+\sum_{x_{k} \in B \backslash C} \alpha_{k}=q ; \\
& \sum_{x_{j} \in B \backslash A} \gamma_{j}+\sum_{x_{k} \in B \backslash C} \alpha_{k}=q ; \\
\sum_{x_{u} \in C} \gamma_{u}= & \sum_{x_{j} \in B \backslash A} \gamma_{j}+\sum_{x_{\ell} \in C \backslash B} \gamma_{\ell}=q,
\end{aligned}
$$

where $q_{1}$ is the number of pairs $(x, y)$ with $x \in A \backslash B, y \in B \backslash A$ and $y<x, q_{2}$ is the number of pairs $(y, z)$ with $y \in B \backslash C, z \in C \backslash B$ and $z<y$, and $q=q_{1}+q_{2}$. Let

$$
\begin{aligned}
h(\mathbf{x}) & =\sum_{x_{s} \in A} \alpha_{s} x_{s}+\sum_{x_{u} \in C} \gamma_{u} x_{u} \\
& =\sum_{x_{i} \in A \backslash B} \alpha_{i} x_{i}+\left(\sum_{x_{j} \in B \backslash A} \gamma_{j} x_{j}+\sum_{x_{k} \in B \backslash C} \alpha_{k} x_{k}\right)+\sum_{x_{\ell} \in C \backslash B} \gamma_{\ell} x_{\ell}
\end{aligned}
$$

and $\mathscr{H}$ the hyperplane of $\mathbb{R}^{d}$ defined by $h(\mathbf{x})=q$. Then $h(\rho(A))=h(\rho(B))=h(\rho(C))=q$. We claim that, for any antichain $D$ of $P$ with $D \neq A, D \neq B$ and $D \neq C$, one has $h(\rho(D))<q$. Let $D=D_{1} \cup D_{2}$ with $D_{1}$ is an antichain of $A \triangle B$ and $D_{2}$ is an antichain of $B \triangle C$. Since $A \triangle B, B \triangle C$ are connected, it follows that $h\left(\rho\left(D_{1}\right)\right)<q_{1}$ and $h\left(\rho\left(D_{2}\right)\right)<q_{2}$. Thus $h(\rho(D))=h\left(\rho\left(D_{1}\right)\right)+h\left(\rho\left(D_{2}\right)\right)<q_{1}+q_{2}=q$.

Case 3. $B \notin A, B \cap A \neq \varnothing, C \notin B, C \cap B \neq \varnothing, C \cap A=\varnothing$, and $P$ has rank 2 .

For each $x_{i} \in P$ we write $c(i)$ for the number of maximal chains, which contain $x_{i}$. Let $q$ be the number of maximal chains in $P$. Since each $x_{i} \in A$ is maximal element and each $x_{k} \in C$ is minimal element, $\sum_{x_{i} \in A} c(i)=\sum_{x_{k} \in C} c(k)=q$. Then

$$
\begin{aligned}
\sum_{x_{j} \in B} c(j) & =\sum_{x_{s} \in B \cap A} c(s)+\sum_{x_{t} \in B \cap C} c(t)+\sum_{x_{u} \in B \backslash(A \cup C)} c(u) \\
& =\sum_{x_{s} \in B \cap A} c(s)+\sum_{x_{t} \in B \cap C} c(t)+\left(\sum_{x_{v} \in A \backslash B} c(v)-\sum_{x_{t} \in B \cap C} c(t)\right) \\
& =\sum_{x_{i} \in A} c(i)=q .
\end{aligned}
$$

Let $h(\mathbf{x})=\sum_{x_{i} \in P} c(i) x_{i}$ and $\mathscr{H}$ the hyperplane of $\mathbb{R}^{d}$ defined by $h(\mathbf{x})=q$. Then $h(\rho(A))=$ $h(\rho(B))=h(\rho(C))=q$. We claim that, for any antichain $D$ of $P$ with $D \neq A, D \neq B$ and $D \neq C$, one has $h(\rho(D))<q . D=A_{1} \cup B_{1} \cup C_{1}$ with $A_{1} \subset A \backslash B, B_{1} \subsetneq B$, and $C_{1} \subsetneq C \backslash B$. Now, we define two subsets of $B$ :

$$
\begin{aligned}
& B_{2}=\left\{x_{j} \in B \mid x_{j}<x_{i}, x_{i} \in A_{1}\right\} \\
& B_{3}=\left\{x_{j} \in B \mid x_{k}<x_{j}, x_{k} \in C_{1}\right\} .
\end{aligned}
$$

Then $B_{1} \cap B_{2}=B_{1} \cap B_{3}=B_{2} \cap B_{3}=\varnothing$ and $B_{1} \cup B_{2} \cup B_{3} \subset B_{3}$. Let $\sum_{x_{i} \in A} c(i)=q_{1}, \sum_{x_{j} \in B_{1}} c(j)=$ $q_{2}, \sum_{x_{k} \in C_{1}} c(k)=q_{3}, \sum_{x_{j} \in B_{2}} c(j)=q_{1}^{\prime}$, and $\sum_{x_{j} \in B_{3}} c(j)=q_{3}^{\prime}$. Since $A \triangle B, B \triangle C$ are connected, it follows that $q_{1}<q_{1}^{\prime}$ and $q_{3}<q_{3}^{\prime}$. Hence

$$
\begin{aligned}
h(\rho(D)) & =\sum_{x_{i} \in A_{1}} c(i)+\sum_{x_{j} \in B_{1}} c(j)+\sum_{x_{k} \in C_{1}} c(k) \\
& =q_{1}+q_{2}+q_{3}<q_{1}^{\prime}+q_{2}+q_{3}^{\prime} \\
& =\sum_{x_{j} \in B_{2}} c(j)+\sum_{x_{j} \in B_{1}} c(j)+\sum_{x_{j} \in B_{3}} c(j) \leqslant \sum_{x_{j} \in B} c(j)=q .
\end{aligned}
$$


Thus $h(\rho(D))<q$.

Case 4. $B \notin A, B \cap A \neq \varnothing, C \cap B=\varnothing$, and $C \cap A=\varnothing$.

Since $P$ has rank 2, we can show $h(\rho(D))<q$ by the same argument in Case 3 (Suppose $C \cap$ $B=\varnothing)$.

Case 5. $B \notin A, B \cap A=\varnothing, C \cap B=\varnothing$ and $C \cap A=\varnothing$.

Since $P$ has rank 2, we can show $h(\rho(D))<q$ by the same argument in Case 3 (Suppose $B \cap A=$ $C \cap B=\varnothing)$.

In conclusion, each $\mathscr{H}$ is a supporting hyperplane of $\mathscr{C}(P)$ and $\mathscr{H} \cap \mathscr{C}(P)$ coincides with the convex hull of $\{\rho(A), \rho(B), \rho(C)\}$, as desired.

Corollary 1. Triangles in 1-skeleton of $\mathscr{O}(P)$ or $\mathscr{C}(P)$ are in one-to-one correspondence with faces of 2-dimensional simplex of each polytope.

Funding: This research received no external funding.

Conflicts of Interest: The author declares no conflict of interest.

\section{References}

1. Stanley, R. Two poset polytopes. Discrete Comput. Geom. 1986, 1, 9-23. [CrossRef]

2. Hibi, T.; Li, N. Unimodular equivalence of order and chain polytopes. Math. Scand. 2016, 118, 5-12. [CrossRef]

3. Hibi, T.; Li, N.; Sahara, Y.; Shikama, A. The numbers of edges of the order polytope and the chain polytope of a finite partially ordered set. Discret. Math. 2017, 340, 991-994. [CrossRef]

4. Hibi, T.; Li, N. Cutting convex polytopes by hyperplanes. Mathematics 2019, 7, 381. [CrossRef] 